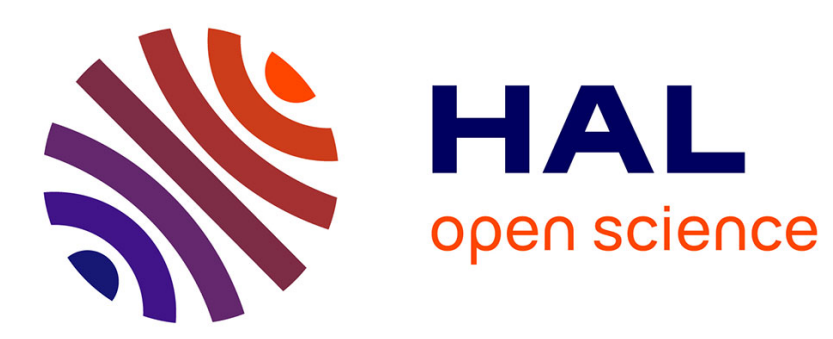

\title{
Genre et ethnicité dans la division du travail en santé : la responsabilité politique des États
}

\author{
Marguerite Cognet
}

\section{To cite this version:}

Marguerite Cognet. Genre et ethnicité dans la division du travail en santé : la responsabilité politique des États. L'Homme et la Société, 2010, 176-177 (2), 10.3917/lhs.176.0101 • ird-01292203

\section{HAL Id: ird-01292203 \\ https://hal.ird.fr/ird-01292203}

Submitted on 22 Mar 2016

HAL is a multi-disciplinary open access archive for the deposit and dissemination of scientific research documents, whether they are published or not. The documents may come from teaching and research institutions in France or abroad, or from public or private research centers.
L'archive ouverte pluridisciplinaire HAL, est destinée au dépôt et à la diffusion de documents scientifiques de niveau recherche, publiés ou non, émanant des établissements d'enseignement et de recherche français ou étrangers, des laboratoires publics ou privés. 


\section{GENRE ET ETHNICITÉ DANS LA DIVISION DU TRAVAIL EN SANTÉ : LA RESPONSABILITÉ POLITIQUE DES ÉTATS} Marguerite Cognet

\section{L'Harmattan | « L'Homme et la société »}

2010/2 nº 176-177 | pages 101 à 129

ISSN 0018-4306

ISBN 9782296139381

\section{Article disponible en ligne à l'adresse :}

http://www.cairn.info/revue-1-homme-et-la-societe-2010-2-page-101.htm

\section{!Pour citer cet article :}

Marguerite Cognet, «Genre et ethnicité dans la division du travail en santé : la responsabilité politique des États », L'Homme et la société 2010/2 (n 176-177), p. 101-129.

DOI 10.3917/lhs.176.0101

Distribution électronique Cairn.info pour L'Harmattan.

(C) L'Harmattan. Tous droits réservés pour tous pays.

La reproduction ou représentation de cet article, notamment par photocopie, n'est autorisée que dans les limites des conditions générales d'utilisation du site ou, le cas échéant, des conditions générales de la licence souscrite par votre établissement. Toute autre reproduction ou représentation, en tout ou partie, sous quelque forme et de quelque manière que ce soit, est interdite sauf accord préalable et écrit de l'éditeur, en dehors des cas prévus par la législation en vigueur en France. Il est précisé que son stockage dans une base de données est également interdit. 


\title{
Genre et ethnicité dans la division du travail en santé : la responsabilité politique des États
}

Marguerite COGNET

\begin{abstract}
«Il y a une dynamique de l'inégalité, au sein de laquelle les migrations du travail sont implantées, qui ne cesse de différencier les pays en importateurs et exportateurs de main-d'œuvre. ${ }^{1}$ »
\end{abstract}

La division du travail en santé par le genre est un phénomène aujour$\mathrm{d}^{\prime}$ hui bien connu ${ }^{2}$. En dépit d'une percée notable des femmes dans les professions médicales - aujourd'hui $39 \%$ de l'ensemble des médecins exerçant dans l'hexagone et $39 \%$ de l'ensemble des spécialistes - qui sont les emplois les plus valorisés du secteur, bénéficient des meilleures conditions de travail (hauteur des rémunérations, autonomie, position hiérarchique) et du plus grand prestige social, elles restent minoritaires dans les spécialisations médicales et chirurgicales les plus en vue. Elles constituent près de $40 \%$ des effectifs des médecins généralistes mais seulement $18 \%$ des spécialistes en cardiologie et $23 \%$ de l'ensemble des spécialités chirurgicales ${ }^{3}$. Les données récentes ${ }^{4}$ ne contredisent toujours pas ce que

1. Saskia SASSEN, La globalisation. Une sociologie (traduit par P. Guglielmina), Paris, Gallimard, 2009, p. 157.

2. Geneviève Cresson, Le travail domestique de santé, Paris, L'Harmattan, 1995 ; Claudine Herzlich, Martine Bungener, Geneviève PAicheler et Marie-Christine Zu$\mathrm{BER}$, Cinquante ans d'exercice de la médecine en France. Carrières et pratiques des médecins français (1930-1980), Paris, CERMES, INSERM-Doin, 1993, p. 273.

3. Daniel SICART, Les médecins. Estimations au $1^{\text {er }}$ janvier 2007, $\mathrm{n}^{\circ} 115$, Paris, Direction de la recherche des études de l'évaluation et des statistiques (DREES), 2007.

4. Ibidem, et du même auteur : Les professions de santé au $1^{\text {er }}$ janvier $2003, \mathrm{n}^{\circ} 52, \mathrm{~Pa}-$ ris, DREES : Bureau professions de santé, 2004 ; et Les professions de santé au $1^{\text {er }}$ janvier 2005, n 82, Paris, DREES : Bureau professions de santé, 2005.

L'homme et la société, $\mathrm{n}^{\circ}$ 176-177, avril-septembre 2010 
soulignaient Claudine Herzlich et ses collaboratrices ${ }^{5}$, à savoir que lorsque les femmes accèdent aux professions médicales, voire y sont majoritaires, c'est sur les domaines les moins spécialisés et où le prestige est moindre comparativement aux spécialités nécessitant des technologies ultra-sophistiquées ${ }^{6}$. À l'inverse, elles restent très largement surreprésentées dans les emplois de subordonnées médicales (infirmières, aides-soignantes) et les métiers socialement les plus disqualifiés des services de santé. Des auteurs soulignent à ce propos l'aggravation de la situation des femmes dans le développement du secteur tertiaire au cours des années quatre-vingt et en particulier avec la création des services à la personne et l'externalisation de certaines tâches, notamment celles liées à l'entretien, considérées comme non directement productives ${ }^{7}$.

Au-delà du genre, quelques travaux ont révélé d'autres dimensions sociales de la discrimination, dont les origines sociales, géographiques, les appartenances ethniques et religieuses ${ }^{8}$. Nos recherches, réalisées en France et au Canada, conduisent à cette même évidence. Quoique la division genrée et ethnicisée ou racisée ${ }^{9}$ du travail soit en général perçue comme une sorte de division spontanée et naturelle du fait de « compétences intrinsèques » qui caractériseraient des groupes d'individus, elle est en réalité le produit de rapports sociaux de domination construits dans une histoire

5. Claudine Herzlich, Martine Bungener, Geneviève PAICHELER et Marie-Christine Zuber, Cinquante ans d'exercice de la médecine en France. Carrières et pratiques des médecins français (1930-1980), op. cit.

6. Daniel SiCART, Les médecins. Estimations au $1^{\text {er }}$ janvier 2007, op. cit.

7. Laurence COUTROT et Annick KIEFFER, Interaction de l'âge et du genre dans le travail peu qualifié, presenté à la $\mathrm{XI}^{\mathrm{e}}$ journée d'analyse longitudinale du marché du travail : "Genre et données longitudinales », Dijon, 27-28 mai 2004.

8. Eliot Freidson, La profession médicale, Paris, Payot, 1984 ; Claudine Herzlich, Martine Bungener, Geneviève PAICHELER et Marie-Christine Zuber, op. cit.; Everett Cherrington HugHes, Men and their Work, Glencoe, The Free Press, 1967 ( $2^{\mathrm{e}}$ éd.) ; David N. SolOMON, «Ethnic and Class Difference among Hospitals as Contingencies in Medical Careers », American Journal of Sociology, 1961, 66(5), p. 412-437.

9. Même si pour la suite du texte, nous parlerons d'ethnicité et d'ethnicisation, nous voulons ici souligner la collusion entre ces deux notions. Si dans le langage courant une assignation ethnique est supposée signifier une différence de pratiques culturelles et une assignation «raciale», une différence inscrite dans le biologique, reliée plus ou moins explicitement à des attributs phénotypiques, et qui revêt de ce fait un caractère intergénérationnel, nous soutenons, à l'instar de Colette Guillaumin (L'idéologie raciste, Paris, Gallimard, 1992 [2 $2^{\mathrm{e}}$ éd. 2002]), que ces constructions sociales de différences procèdent de la racisation des individus qui n'est autre que l'instruction d'une altérité radicale par essentialisation de toutes sortes de traits culturels ou biologiques attribués, nolens volens, à l'ordre de la nature des choses. 
séculaire et régénérés par un contexte économique, politique et social d'un monde globalisé où s'accroissent les interdépendances ${ }^{10}$.

Nous reviendrons ici sur les résultats d'études que nous menons depuis les années quatre-vingt-dix en France et au Canada. En mettant ces terrains en perspective, notre objectif vise moins une comparaison terme à terme entre les deux pays qu'explorer ce que ces terrains ont en commun, relativement à la gestion des ressources humaines dans un contexte de rationalisation des coûts de santé. À partir de l'observation de la place des femmes et des migrants dans l'organisation contemporaine des soins et des services de santé nous voulons explorer le rôle de l'État et des logiques capitalistes, examiner l'impact des politiques publiques sous-tendues par ces logiques sur la division du travail en santé. À l'instar de nombre d'auteurs, nous considérons que les catégories de genre, entendu comme l'attribution sociale des rôles des sexes, et d'ethnicité, entendue comme attribution sociale des rôles des filiations supposées, pas plus que la classe sociale ne constituent des catégories objectives. Bien au contraire, genre, ethnicité et classe ne sont que les produits de constructions sociales par la mise en exergue de caractéristiques chargées d'emblématiser de soidisant différences entre des groupes d'individus et de les réifier comme minorités naturelles ${ }^{11}$. Ces catégories, une fois construites et essentialisées, agissent comme prophétie autoréalisatrice ${ }^{12}$, et s'imposent comme autant d'injonctions de conformité aux individus qui en reçoivent les rôles.

Si l'organisation genrée et ethnicisée des soins et des services n'est ni nouvelle, ni ponctuelle, il nous semble qu'aujourd'hui les objectifs de rationalisation des coûts de santé favorisent une combinaison de dimension internationale qui sous-tend la migration des femmes qui ne sont plus seulement des « rejoignantes » mais aussi des femmes qui migrent seules, caractéristique des migrations internationales contemporaines ${ }^{13}$. Entamée

10. Saskia SASSEN, La globalisation. Une sociologie, op. cit.

11. Elsa DORLIN, «Dark care : de la servitude à la sollicitude », in Patricia PAPERMAN et Sandrine LAUGIER (éds.), Le souci des autres. Éthique et politique du care, Paris, EHESS, 2005, p. 87-97; Elsa DorLIN, Sexe, genre et sexualités. Introduction à la théorie féministe, 2008, Paris, PUF ; Colette GuILLAUMIN, «Une société en ordre. De quelquesunes des formes de l'idéologie raciste », Sociologie et sociétés, XXIV(2), 1992, p. 13-23 ; Colette Guillaumin, L'idéologie raciste, op. cit.

12. Robert King Merton, Éléments de théorie et de méthode sociologique, Paris, Armand Colin, 1997 ( $3^{\mathrm{e}}$ éd.) ; William I. Thomas \& Dorothy Swaine ThomAs, The Child in America : Behavior Problems And Programs, New York, Alfred A. Knopf, 1928.

13. Asuncion FRESNOZA-FLOT, Migration, genre et famille transnationale : l'exemple des mères migrantes philippines en France, Doctorat de sociologie, Université Paris VII, Paris, 2008 ; Asuncion FRESNOZA-FLOT, Les migrantes philippines en France : mère ou nourrice, l'obligation de choisir?, presenté à « Migrations humaines et circulations des 
dans le courant des années quatre-vingt, la gestion rationalisée des coûts de santé, qui passait par un retrait des fonds publics, s'est traduite, entre autres, par une réduction des lits d'hospitalisation avec une externalisation de certains services et soins - notamment en ce qui a trait aux services et soins aux polyhandicapés, aux personnes âgées semi-dépendantes et dépendantes ou atteintes de maladies chroniques invalidantes - , une modification des statuts d'emploi au sein des services publics et un recours à la sous-traitance. Nous montrerons que dans ces deux sociétés, en dépit de leurs différences, les femmes, a fortiori les femmes ethnicisées - qu'elles soient migrantes ou supposées l'être via leurs ascendances - et, parmi elles, notamment celles qui sont cataloguées comme originaires de pays du Sud, payent un lourd tribut dans le cours de ces réformes. Cette « redivision » du travail passe à la fois par les politiques sanitaires et les réformes qui en découlent et par les politiques migratoires visant à pallier les besoins de main-d'œuvre. À ce titre, la situation canadienne vaut pour exemple de ce qui se profile en France en matière de soins et de services à la personne ${ }^{14}$ et que d'ailleurs, en substance, confirment des indicateurs récents en termes d'externalisation et de développement du secteur pri$v^{1}{ }^{15}$. L'étude de l'organisation du travail en santé dévoile des points d'intersection entre ethnicité, classe et genre qui poussent les femmes, en particulier les migrantes ou issues de parents immigrés, vers certains emplois et les tiennent à l'écart de certains autres. Un résultat qui converge avec d'autres travaux ${ }^{16}$.

ressources », «La santé en temps de mondialisation », Paris, 24-25 septembre 2009; Éléonore KoFMAN, «Genre, migrations, reproduction sociale et Welfare State », Cahiers du CEDREF : Femmes, Genre, Migrations et Mondialisation: Un état des problématiques, 2008, p. 101-124 ; Mirjana MorOKVASIC, « Femmes et genre dans l'étude des migrations internationales », Cahiers du CEDREF, ibidem, p. 33-56.

14. Aujourd'hui, en France, selon la convention nationale pour le développement des services à la personne, signée le 22/11/04, ces services recouvrent un ensemble de services dont les services à la famille et les soins et des services à domicile.

15. Alexandra CHOL, «Les emplois familiaux en 2004 : un secteur en forte croissance », Premières synthèses, DARES, 2006, 19, 1(1), 6 p., cf. http://www.travailsolidarite.gouv.fr/IMG/pdf/2006.05-19.1.pdf ; Alexandra CHOL, «Les services à la personne en 2005 : poussée des entreprises privées », Premières synthèses, DARES, 2007, 20(1), 7 p., cf. http://www.travail.gouv.fr/IMG/pdf/2007.05-20.1.pdf ; François-Xavier DEVETTER, Florence JANY-CATRICE et Thierry RIBAULT, Les services à la personne, Paris, La Découverte, 2009.

16. Pat Armstrong \& Hugh Armstrong, Wasting Away. The Undermining of Canadian Health Care (vol. 1), Toronto, Oxford University Press, 2003 ; Jane ARONSON \& Sheila M. Neysmith, « The Work of Visiting Homemakers in Context of Cutting in Long Term Care », Canadian Journal of Public Health, 1996, 87(6), p. 422-425 ; Myriam BALs, Les domestiques étrangères au Canada, esclaves de l'espoir, Paris, Montréal, L'Harmat- 
Notre propos suivra une argumentation en quatre points. Après quelques précisions relatives à la démarche méthodologique conduite dans les recherches, nous reprendrons de façon synthétique les analyses qui, sur le terrain français comme sur le terrain canadien, révèlent une stratification des emplois par le genre et les origines. Dans un troisième point, nous montrerons en quoi ces analyses ont à voir avec les réformes dans les politiques de santé. Mais nous verrons que la seule lecture des politiques sanitaires n'est pas suffisante pour comprendre le processus qui structure les emplois par le genre et la «race». D'autres politiques doivent venir s'articuler à celles-ci : les politiques de l'emploi bien sûr, mais également les politiques migratoires et toutes les formes de sélection qu'elles autorisent. Ce sera le quatrième point.

\section{Encadré méthodologique \\ Données d'enquêtes en France et au Canada}

Nos enquêtes sur le terrain français ont commencé à la fin des années quatrevingt-dix. La première recherche a été réalisée dans un Établissement public de santé mentale en région parisienne ${ }^{17}$. Les données reprises ici sont extraites de l'analyse des fichiers des ressources humaines. Nous disposions de variables sociodémographiques et socioprofessionnelles. Ces données ont été traitées sur SADE et EXCEL (analyses uni- et multivariées). Les analyses statistiques ont été complétées par des entretiens semi-directifs $(\mathrm{n}=65)$ et des observations in situ. Quoique l'évolution de l'organisation sanitaire psychiatrique présente des différences importantes en regard de celle des soins généraux de par l'histoire de ses institutions, cela n'atténue pas la portée des données traitées ici dans la mesure où les réformes hospitalières menées sous les auspices de la rationalisation des coûts se sont traduites en priorité par une forte injonction de réduction des lits, la mise en place

tan, 1999 ; Sabah Снаїв, «Femmes immigrées et travail salarié en France », Les Cahiers du CEDREF : Femmes, genre, migrations et mondialisation, op. cit., p. 209-230 ; Éléonore KoFMAN, «Genre, migrations, reproduction sociale et Welfare State », op. cit.; Sheila M. NeYSMITH \& Jane ARONSON, «Working Conditions in Home Care : Negotiating Race and Class Boundaries in Gendered Work », International Journal of Health Services, 1998, (27), 3 ; Francesca SCRINZI, « Consumi culturali. I processi di etnicizzazione delle donne marocchine a Genova », in Andrea TORRE (éd.), Non sono venuta a scoprire le scarpe. Voci di donne immigrate in Liguria, Cuneo, Edizioni Sensibili alle foglie, 2001, p. 63-89; et du même auteur : La marchandisation globalisée du travail domestique ou les femmes migrantes et la néodomesticité, (présenté au colloque international des recherches féministes francophones, 2002) ; «Les employées de maison migrantes en Italie. Ethnicisation et contrôle dans le circuit catholique à Gênes », Canadian Women Studies, 2003, 22(3/4), p. 182-187 ; Les migrantes dans les emplois domestiques en France et en Italie. Construction sociale de la relation de service au croisement des rapports sociaux de sexe, de race et de classe, Doctorat de sociologie, Paris VII Diderot, Paris, 2005.

17. Marguerite COGNET, Migrations, groupes d'origines et trajectoires : vers une ethnicisation des rapports socioprofessionnels?, Villeneuve d'Ascq, Presses universitaires du Septentrion, 1999. 
d'alternatives aux hospitalisations et diverses formes de sous-traitance au secteur privé. Dans ce sens, nous pouvons considérer que sur les points qui nous importent dans cet article, la réforme des hôpitaux psychiatriques au cours des années quatre-vingt fut un signe précurseur d'un mouvement d'ensemble du système sanitaire que nous observons aujourd'hui dans une étude en cours sur les soins et services à la personne - un mouvement que la France partage avec l'ensemble des pays européens et, au-delà, avec le monde occidental.

Dans le cadre de notre recherche actuelle sur les soins et services à la personne en Île-de-France, nous avons analysé les différents textes ayant trait aux soins proprement dits et aux services domestiques. Pour les soins, il s'agit notamment des textes qui, depuis 2002 (loi du 2 janvier 2002 rénovant l'action sociale et médico-sociale), procèdent à la restructuration des missions sanitaires avec la délégation d'une partie des soins et des services vers le secteur désormais nommé «médico-social». Cette réforme s'articule avec celle de l'organisation des services à domicile, en particulier avec l'adoption du $1^{\text {er }}$ plan Borloo en 2005 qui vise à développer ce secteur (mise en œuvre de l'agence nationale des services à la personne ; soutien au programme mobilité-emploi de France Terre d'Asile, etc.) Ces analyses sont triangulées avec des données collectées en entretien (collecte en cours) auprès de femmes exerçant dans ces secteurs (travail déclaré de gré à gré ou via des agences ; travail non déclaré de gré à gré). Ces données sont également confrontées à des observations in situ de travaux d'étudiantes de master et de doctorat de sociologie et anthropologie que nous encadrons.

Le terrain canadien explore les effets de la réforme du système de santé telle qu'elle s'est déclinée au Québec et que traduit bien la notion de virage ambulatoire $^{18}$. Nous ferons ici référence en particulier à une étude que nous avons réalisée en 2000 à Montréal concernant les conditions de travail d'auxiliaires familiaux, catégorie d'emploi en forte augmentation suite à la réforme du système de santé et l'externalisation des soins et services à la personne. Nous reprendrons plus spécifiquement les résultats d'un questionnaire réalisé sur un échantillon de 180 individus exerçant auprès de la clientèle des services de maintien à domicile des Centres locaux de services communautaires ${ }^{19}$ (CLSC), qu'ils soient leurs propres employés ou ceux d'agences privées avec lesquelles les CLSC sous-traitent.

18. Denyse Côté, Éric Gagnon, Claude Gilbert, Nancy Guberman, Francine SAILLANT, Nicole Thivierge, Marielle Tremblay et al., Qui donnera les soins? Les incidences du virage ambulatoire et des mesures d'économie sociale sur les femmes du Québec, Ottawa, Condition féminine Canada, 1998 ; Denyse CôTÉ et Guilhème PÉrodEAU, Le Virage Ambulatoire. Défis et enjeux, PUQ, 2002 ; Éric GAGNON, Nancy GuBERMAN, Denyse Côté, Claude GiLbert, Nicole ThIVIERge et Marielle Temblay, Les impacts $d u$ virage ambulatoire : responsabilités et encadrement dans la dispensation des soins à domicile, Beauport, Direction de santé publique, Régie régionale de la santé et des services sociaux de Québec, 2001 ; Paul-André LAPOINTE, Malie ChAYER, François Bernard MALO et Linda RIVARD, «La reconfiguration des soins de santé et la réorganisation du travail infirmier », Nouvelles pratiques sociales, 2000, 13(2), p. 164-180 ; D. HÉBERT, «Pour contrer la privatisation des services à domicile, accroître les ressources allouées aux CLSC », Fédération de la santé et des services sociaux CSN, 1999 :

http://www.csn.qc.ca/Pageshtml16/CLSCCollCSNdh.html.

19. Les CLSC, sont des structures de santé publique offrant tous les soins et services de santé primaire. 
Indépendamment de la structure qui les emploie (CLSC ou agence), le travail des auxiliaires est défini par des tâches concrètes destinées à assister les personnes en situation de dépendance et semi-dépendance dans l'exécution des activités de la vie quotidienne et domestique. Le corpus de ces données a été traité sur SPSS (analyses uni- et multivariées). Une quarantaine d'entretiens semi-directifs et des observations in situ ont permis d'approfondir les trajectoires sociales et professionnelles des auxiliaires.

\section{Le constat empirique}

Dans la première étude, l'analyse statistique de la répartition des genres dans l'hôpital français attestait de la division traditionnelle des sexes. Les hommes, majoritaires dans les emplois de médecins, de cadres infirmiers supérieurs ${ }^{20}$, voyaient leurs effectifs décroître au fur et à mesure que l'on descendait la ligne hiérarchique et la valeur symbolique des emplois et ce, de façon très significative $(\mathrm{p}=0.00)$. On notait une inversion des courbes des taux entre hommes et femmes alors que les conditions de travail (rémunération, autonomie, type de tâches) liées aux différentes catégories d'emplois se dégradaient. L'étude des profils accusait également des écarts de répartition selon les lieux de naissance. Les nationaux occupaient de meilleures situations que les immigrants. Au-delà, une dimension ethnoraciale structurait une ligne de partage des emplois : a) $1 / 3$ des Domiens occupaient des postes d'aides-soignants et agents de service hospitalier ; $0.9 \%$ des Français nés en métropole étaient dans ce cas, b) $76 \%$ des Sud-Américains étaient médecins alors que ce taux était à peine de $21 \%$ pour les Maghrébins et $10 \%$ pour les Africains. Les 2/3 des femmes originaires du Maghreb étaient dans les catégories C ou D. Toutes les femmes d'Afrique sub-saharienne et $50 \%$ des hommes étaient dans la catégorie $\mathrm{D}^{21}$.

L'accroissement des soins infirmiers à domicile dans les années 19801990 où les soins de nursing assurés par les aides-soignantes sont devenus prépondérants ${ }^{22}$, la réorganisation du travail hospitalier, la sous-traitance

20. L'importance des hommes dans l'encadrement infirmier est particulière à la psychiatrie même si cette tendance s'est progressivement inversée dans la décennie 19902000. En 2005, à l'échelle nationale, les cadres infirmiers en psychiatrie comptaient $48 \%$ d'hommes (Daniel SICART, Les professions de santé au $1^{\text {er }}$ janvier 2005, op. cit.). Dans les soins généraux, les hommes ne sont majoritaires que dans les emplois de médecins.

21. Marguerite COGNET, Migrations, groupes d'origines et trajectoires : vers une ethnicisation des rapports socioprofessionnels?, op. cit.

22. Sophie BRESSÉ, Les services de soins infirmiers à domicile (Ssiad) et l'offre de soins infirmiers aux personnes âgées en 2002, cf. http://www.sante.gouv.fr/drees/etuderesultat/er-pdf/er350.pdf, 2004. Sophie Bressé rapporte que depuis 1980, l'offre des soins et services infirmiers à domicile a été multipliée par 20. 
en maison d'accueil spécialisée (MAS) ${ }^{23}$ où vont se développer des emplois d'aide-médico-psychologique (AMP) ${ }^{24}$, préfiguraient bien l'organisation qui s'étend à l'ensemble du système de santé primaire entraînant l'éclosion d'une myriade de nouvelles catégories d'emplois à la lisière du système hospitalier - auxiliaires de vie sociale qui œuvrent à domicile auprès des personnes dépendantes et semi-dépendantes, employés familiaux polyvalents, assistants de vie, soit toutes sortes d'emplois peu qualifiés dans les services à la personne - où nous retrouverons surtout des femmes issues des classes populaires ou/et de l'immigration ${ }^{25}$. Parmi ces dernières catégories on relève les emplois d'assistantes familiales créés au milieu des années 1980-1990. Recrutées comme assistantes familiales par l'hôpital, voire par un système associatif en son sein, ces femmes se voient confier la garde d'un patient dont elles assureront les besoins fondamentaux 24 heures sur 24, 7 jours sur 7 . Ces postes sont en totalité occupés par des femmes et, parmi elles, $46 \%$ sont nées dans un pays d'Afrique ou dans les DOM. La rémunération est calculée sur la base de $5 \mathrm{~h} / \mathrm{j}$ au taux du SMIC. Cette catégorie d'emploi, frappée d'invisibilité et habituellement

23. Les MAS ne sont d'ailleurs qu'une part infime du parc de l'organisation sanitaire sous-traitée au privé. Depuis les années quatre-vingt, le nombre de structures destinées non seulement aux populations psychiatriques chroniques mais également aux populations handicapées, dépendantes, semi-dépendantes, atteintes de maladies chroniques invalidantes, ne cesse de croître (foyer de vie, maison médicalisée, foyer d'accueil, etc.).

$24.86 \%$ des AMP travaillent dans le secteur privé associatif. La très grande majorité sont des femmes, et, parmi elles, nombreuses sont immigrées ou filles d'immigrés. Les tâches des AMP en France relèvent de l'assistance dans les gestes de la vie quotidienne des personnes dépendantes ou semi-dépendantes). La rémunération a toujours été proche du SMIG.

25. Christelle AVRIL, «Quel lien entre travail et classe sociale pour les travailleuses du bas de l'échelle ? L'exemple des aides à domicile auprès des personnes âgées dépendantes », Lien social et Politiques : RIAC(49), 2003, p. 147-154 ; Lise CAUSSE, Chantal LABRUYÈRE et Christine FOURNIER, Les aides à domicile. Des emplois en plein remueménage, Paris, La Découverte et Syros, 1998 ; Sabah CHAїв, «Femmes immigrées et travail salarié en France », op. cit.; Alexandra CHOL, "Les emplois familiaux en 2004 : un secteur en forte croissance », op. cit.; Geneviève CRESSON, Le travail domestique de santé, op. cit.; François-Xavier DEVETTER, Florence JANY-CATRICE et Thierry RIBAULT, Les services à la personne, op. cit.; Elsa DoRLIN, « Dark care : de la servitude à la sollicitude », op. cit.; Annie Dussuet, «Les emplois familiaux, une forme "féminine" de précarité », in Marion Del Sol, Anne Eydoux, Annie Gouzien, Pierre Merle et Pascale TURQuET (éds.), Nouvelles dimensions de la précarité, Rennes, Presses universitaires de Rennes, 2001, p. 53-66 ; Nicole GADREY, Travail et Genre. Approches croisées, Paris, L'Harmattan, 2001 ; Odile MERCKLING, «L'emploi des femmes étrangères et issues de l'immigration », Hommes \& Migrations, 2002 (1239), p. 100-111 ; Odile MercKLING, Emploi, migration et genre. Des années 1950 aux années 1990, Paris, L'Harmattan, 2003. 
ignorée dans les calculs du personnel hospitalier, constitue un indicateur de l'externalisation de certains soins à la marge du marché du travail.

Dans le groupe des auxiliaires québécois, statutairement au plus bas de la hiérarchie soignante, on ne s'étonnera pas de trouver majoritairement des femmes $(80 \%)$. On s'étonnera peut-être davantage du pourcentage d'immigrants : $47 \%$ quand, pour la même période, leur taux dans la population totale de Montréal comptait pour moitié (26\%). La comparaison entre les deux types d'employeur montre que les immigrants ont une plus forte probabilité d'être employés par une agence privée que par un CLSC $(\mathrm{p}=0.002) .53 \%$ déclarent être employés par une agence contre $30 \% \mathrm{chez}$ les non-immigrants. L'écart entre les deux types d'employeur ne vaut cependant pas pour tous les immigrants. Comme en France, une analyse plus fine révèle une division « raciale » du travail. La régression logistique confirme un effet net de cette dimension sur la distribution entre les deux types d'employeur. La probabilité d'accéder à un emploi en CLSC pour les noirs apparaît significativement réduite par rapport aux blancs (coefficient estimé $B=-1.24$ pour un seuil très significatif $p=0.01$ ). Après contrôle des variables confondantes (niveau d'éducation, formation au métier, compétences linguistiques), l'effet de la couleur de peau reste très significatif ( $\mathrm{p}=0.01, \mathrm{~B}=-1.17)$, attestant d'une plus grande difficulté d'accès aux emplois en CLSC pour les noirs que pour les blancs ${ }^{26}$. Or, être employée d'un CLSC ou d'une agence est, pour une auxiliaire familiale, très différent sur nombre de points même si le contenu des soins et services, lui, ne varie pas $^{27}$.

Au moment de l'enquête, une auxiliaire familiale montréalaise, employée en CLSC perçoit $15 \$ / \mathrm{h}$, soit deux fois le salaire horaire de son homologue d'agence privée dont le salaire horaire moyen de $7,10 \$$ avoisine le salaire minimum $(7 \$ / \mathrm{h})$. Les hommes, plus difficiles à recruter, sont mieux payés, leur salaire peut atteindre $11 \$ / \mathrm{h}$ quand celui des femmes

26. Marguerite COGNET, «L'accès à l'emploi dans les services de santé : l'effet de la dimension ethnique. Étude du groupe des auxiliaires familiaux et préposés aux bénéficiaires à Montréal », in Jean RENAUD, Annick GERMAIN et Xavier LELOUP (éds.), Racisme et discrimination : permanence et résurgence d'un phénomène inavouable, Québec, Presses de l'université Laval, 2004, p. 60-86.

27. ACSSC, Canada Home Care Labour Market Study, Ottawa, Association canadienne des soins et services communautaires, 1995 ; ACSSC, Health Promotion and the Home Support Worker, Ottawa, Association canadienne des soins et services communautaires, 1995 ; Margaret MACADAM \& Groupe chargé des politiques au Développement des soins à domicile, d. P. C., Myra Conway, Heather Davis, Carol Donovan, Mireille DuMONT-LEMASSON et Sue MORRISON, Les questions relatives aux ressources humaines dans le secteur des soins à domicile au Canada: Point de vue stratégique, Ottawa, Santé Canada, 1999. 
peut descendre à $6 \$ / \mathrm{h}$. Une auxiliaire, employée du CLSC, bénéficie d'un certain nombre d'avantages sociaux, dispose d'un accès à un emploi permanent, d'une perspective de carrière avec prise en considération de son ancienneté, d'un accès à la formation continue, d'un encadrement clinique et d'un recours syndical. La situation des employées du secteur privé s'oppose en tous points à ce modèle, à commencer par la précarité (intérim), l'absence de tout avancement de carrière et celui de tout support syndical. L'encadrement professionnel, la formation et le soutien clinique sont limités quand ils ne sont pas inexistants.

Ce que nous observons aujourd'hui en France montre, à certains signes, que la situation pourrait évoluer de façon similaire, aggravant la discrimination à l'endroit de certaines femmes. Les enquêtes emploi postérieures au tournant du siècle, confirment que non seulement les domaines de l'éducation, de la santé et de l'action sociale restent dévolus aux femmes ${ }^{28}$ mais que, parmi elles, les immigrées et leurs filles sont particulièrement surreprésentées. En 2007, parmi l'ensemble des immigrées actives, six sur dix exerçaient comme employées des services - notamment dans les soins et services aux particuliers où elles comptent pour près d'un quart, alors que c'est seulement le cas d'une française sur dix ${ }^{29}$. Près de $40 \%$ des employées de maison et $20 \%$ des aides à domicile ont un père ou une mère étrangère ${ }^{30}$. Néanmoins, ce ne sont pas toutes les filles d'immigrés qui se retrouvent surreprésentées ici. S'il reste toujours difficile, en France, d'appréhender de manière statistique la répartition des emplois entre «Français descendants de parents d'origine française » et «Français descendants de parents d'origine étrangère» ou encore entre les origines de ces derniers, toutes nos observations, depuis les années 1990, montrent que les plus nombreuses dans le secteur des soins et services à la person-

28. Le personnel des services directs aux salariés compte $86 \%$ de femmes. Pour la seule part des services à domicile le taux de femmes est de $99 \%$ (Annie DussuET, « Les emplois familiaux, une forme "féminine" de précarité », op. cit.; Annie DussuET, Travaux de femmes. Enquêtes sur les services à domicile, Paris, L'Harmattan, 2005). Cf. également François ChEVAliER, Isabelle MACARIO-RAT et Anne MANSUY, Une photographie du marché du travail en 2007. Résultats de l'enquête Emploi, INSEE Première, 2008 (1206), 4.

29. INSEE, «Répartition des actifs occupés immigrés selon le sexe et la catégorie socioprofessionnelle. Enquête Emploi du $1^{\mathrm{er}}$ au $4^{\mathrm{e}}$ trimestre 2007. France métropolitaine, actifs occupés immigrés de 15 ans et plus. Résultats en moyenne annuelle », 2007 ; Jacqueline PERRIN-HAYNES, L'activité des immigrés en 2007, INSEE Première, 2008 (1212), 4 ; Chloé TAVAN, «Les immigrés en France : une situation qui évolue », INSEE Première, 2005 (1042), 4.

30. François-Xavier DevetTer, Florence JANY-CATRICE et Thierry Ribault, Les services à la personne, op. cit. 
ne sont les filles d'originaires du Maghreb. Il semble que les descendantes de migrants venus d'Espagne ou du Portugal dont les femmes étaient très largement employées du secteur domestique, aient davantage bénéficié de l'ascenseur social que les filles de parents algériens ou marocains. Ces observations rejoignent d'autres études qui montrent une plus grande discrimination pour les Françaises descendantes de parents venus de pays colonisés, en particulier du Maghreb et d'Afrique sub-saharienne ${ }^{31}$ et le durcissement de la ligne de partage entre ressortissants des pays européens et des pays tiers pourrait encore l'accentuer ${ }^{32}$.

Le travail des soins et des services à autrui, qu'il soit salarié, de gré à gré ou accompli bénévolement, est un secteur totalement féminisé et largement ethnicisé, ce qui revient à dire que les compétences qu'il requiert sont renvoyées à l'ordre de la nature des femmes quand ce n'est pas à leur culture, à leurs soi-disant aptitudes particulières à en accomplir les tâches ${ }^{33}$. Ce faisant, comme tout marché « féminisé », il fait l'objet d'un discrédit social qui se traduit par une représentation d'un travail non qualifié et donc pouvant prétendre à certaines conditions de reconnaissance dont celles de la rémunération ${ }^{34}$. Ce qu'Elsa Dorlin décrit comme « l'entrecroisement des préjugés sexistes et racistes », assigne ces femmes à ces

31. Dominique Meurs et Ariane PAILHÉ, «Descendantes d'immigrés en France : une double vulnérabilité sur le marché du travail ? », Travail, genre et sociétés, 2008, 20(2), p. 87-107 ; Dominique Meurs, Ariane PAILHÉ et Patrick Simon, «Mobilité entre générations d'immigration et persistance des inégalités : l'accès à l'emploi des immigrés et de leurs descendants en France », Population, 2006, (5/6), p. 763-801 ; Ariane PAILHÉ, « La combinaison des discriminations racistes et sexistes dans l'accès à l'emploi des descendantes d'émigrés maghrébins en France », Nouvelles questions féministes, 2008, 27(1), 92-112 ; Laurence ROUlLEAU-BERGER (éd.), Femmes d'origine étrangère. Travail, accès à l'emploi, discrimination de genre, Paris, Fasild/La Documentation Française, 2004.

32. Andrea RÉA (éd.), Immigration et racisme en Europe, Bruxelles, Éditions Complexe, 1999 ; Francesca SCRINZI, «Quelques notions pour penser l'articulation des rapports sociaux de "race" de classe et de sexe », Cahiers du CEDREF, Femmes, genre, migrations et mondialisation, 2008, p. 81-99.

33. Nicole GADREY, Travail et Genre. Approches croisées, op. cit.; Francesca SCRIN$\mathrm{ZI}$, «Les migrantes dans les emplois domestiques en France et en Italie. Construction sociale de la relation de service au croisement des rapports sociaux de sexe, de race et de classe », Faire Savoirs : L'ethnicisation et la racisation des rapports sociaux en question, 2007 (6), p. 91-98 ; Francesca SCRINZI, « Quelques notions pour penser l'articulation des rapports sociaux de "race" de classe et de sexe », op. cit.

34. Nicole GADREY, Hommes et femmes au travail. Inégalités, différences, identités, Paris, L'Harmattan, 2000 ; Nicole GADREY, Travail et Genre. Approches croisées, op. cit.; Danièle KeRGOAT, «Penser la différence des sexes. Rapports sociaux et division du travail entre les sexes », in Margaret MARUANI (éd.), Femmes, genre et sociétés, Paris, La Découverte, 2005, p. 94-101. 
emplois au nom d'une «prédisposition prétendument naturelle ou culturelle $^{35} \gg$. La division genrée et ethnicisée du travail du «care » contribue à renforcer les représentations naturalisantes des compétences de ces femmes qui, à leur tour, légitiment le recours à leur recrutement pour l'accomplir, rendant ainsi invisible le processus d'exploitation et de discrimination.

Tandis que Jean-Louis Borloo, signataire de la loi du 26 juillet 2005 relative au développement des services à la personne, se félicite de cette manne d'emplois non-délocalisables, nous cherchons à saisir le processus qui organise et pérennise le confinement de ces femmes dans ces emplois dans l'ensemble historicisé des politiques sanitaires, en ce qu'elles structurent et hiérarchisent les emplois, et des politiques migratoires qui déterminent la circulation des femmes. Autrement dit, nous considérons que ce que nous observons de cette division genrée et ethnicisée ne procède pas d'abord de choix individuels des femmes mais bien plutôt des seules possibilités auxquelles elles peuvent accéder dans un univers contraignant, structuré par les politiques.

\section{Les réformes de santé. Le jeu politique des États}

En France et au Québec, la division du travail en santé doit être appréhendée à l'aune des restructurations engagées qui impliquent des réorganisations des soins et services mais également, et de façon liée, une réorganisation des groupes professionnels avec une re-division des tâches ${ }^{36}$. Ce sont également des moments où les valeurs attachées aux différentes tâches sont réévaluées, à la fois financièrement (combien l'État, la société sont prêts à payer pour elles) et symboliquement (le prestige qu'elles confèrent à celui qui les exécute) à la hausse ou à la baisse ${ }^{37}$. C'est sans doute

35. Elsa DORLIN, « Dark care : de la servitude à la sollicitude », op. cit.

36. Yves Buin, Psychiatries : l'utopie, le déclin, Paris, Erès, 1999; Lise Causse, Chantal LABRUYÈre et Christine FOURNIER, Les aides à domicile. Des emplois en plein remue-ménage, op. cit.; Denyse Côté et al., Qui donnera les soins? Les incidences du virage ambulatoire et des mesures d'économie sociale sur les femmes du Québec, op. cit.; Denyse Côté et Guilhème PÉrodeAu, Le Virage Ambulatoire. Défis et enjeux, op. cit.

37. Marguerite COGNET, La compétition aux compétences : le jeu des cultures, présenté au colloque annuel international du Comité de recherche 13 de l'Association des sociologues de langue française (AISLF), « Coopérations, conflits et concurrences dans le système de santé », Centre d'études des rationalités et des savoirs (CERS), université de Toulouse-Le Mirail, 29 et 30 novembre 2001 ; Marguerite COGNET et Laurent RAIGNEAU, "Le genre et l'ethnicité : les critères voilés de la délégation des actes en santé », Cahiers du GRES, Centre d'études ethniques des universités montréalaises, 2002, 3(1), p. 25-38 ; Geneviève CRESSON, «Les soins profanes et la division du travail entre hommes et femmes », in Pierre AÏACH, Dominique CÈBE, Geneviève CRESSON et Claudine PHILIPPE (éds.), 
une des particularités des catégorisations genrées et ethnicisées que de faire l'objet d'une mise en discours qui fait passer les différences construites dans les rapports sociaux pour des différences tenant à la nature et à l'ordre des choses. Néanmoins, dire que l'organisation genrée et ethnicisée du travail de la santé est le produit articulé et historiquement construit des rapports de domination, n'explique pas le processus par lequel ces divisions se reproduisent concrètement. Le recrutement des étrangers et la régression des positions en emploi des Domiens dans l'hôpital, le recours massif aux immigrantes pour les emplois d'aides-soignantes, d'AMP, d'auxiliaires de vie, etc. en France, ou pour les emplois d'auxiliaires au Québec, destinés à assurer les soins aux patients dépendants, sont en réalité autant de conséquences des choix politiques et économiques retenus. Dans les faits, il nous semble qu'il y a une conjonction forte entre la mise en œuvre d'une gestion plus économe du travail en santé et l'affectation de certains individus à certains postes. À l'instar d'autres auteurs, nous interrogeons donc la part que prennent les politiques publiques dans la division du travail ${ }^{38}$, tant dans ce qu'elles initient dans l'organisation des soins de santé et la gestion comptable de ceux-ci, y compris via leur externalisation $^{39}$, que dans celle de l'organisation de l'immigration de travail et de gestion des populations migrantes qui s'ensuit ${ }^{40}$.

Femmes et hommes dans le champ de la santé. Approches sociologiques, Rennes, ENSP, 2001, p. 303-328 ; Francine SAILLANT, «La part des femmes dans les soins de santé », Revue internationale d'action communautaire, 1992, 28(66), p. 95-106.

38. Lise CAusse, Chantal LABRUYÈre et Christine FourniER, Les aides à domicile. Des emplois en plein remue-ménage, op. cit.; Denyse CôTÉ et al., Qui donnera les soins? Les incidences du virage ambulatoire et des mesures d'économie sociale sur les femmes du Québec, op. cit.; Nicole GADrey, Travail et Genre. Approches croisées, op. cit.; Arlette GAUTIER et Jacqueline HeInEN, Le sexe des politiques sociales, Paris, Côté-Femmes, 1993 ; Nancy Guberman, Pierre MAHEU et Chantal MaILlÉ, Et si l'amour ne suffisait pas... Femmes, familles et adultes dépendants, Montréal, Les Éditions du remue-ménage, 1993

39. Cf. le Plan Borloo sur le développement des services à la personne de 2005 (Loi du 26/07/05; décret du 26/12/05). Cf. également Guilhem BENTOGLIO, Développer l'offre de services à la personne, 2005, $\mathrm{cf}$. :

http://lesrapports.ladocumentationfrancaise.fr/BRP/054000383/0000.pdf.

40. Éléonore KOFMAN, «Genre, migrations, reproduction sociale et Welfare State », $o p$. cit.; Claudie LESSELIER, « Politiques d'immigration en France : appréhender la dimension de genre », Les Cahiers du CEDREF : Femmes, Genre, Migrations et Mondialisation. Un état des problématiques, op. cit., p. 190-208 ; Marie-Hélène VALLÉE, « L'immigration féminine au Canada durant les années 1920 : la mise en place d'une politique fondée sur le “genre" », Recherches Féministes, 2002, 15(2), p. 65-85. 


\subsection{Les réformes dans le système de santé français dans les années postcoloniales}

Le recrutement des étrangers et l'évolution des carrières des Domiens sont conjecturels de deux temps forts qui marquent l'évolution de la psychiatrie française sur les quarante dernières années. Le premier concerne la réforme des asiles qui s'est mise en place dans les années soixante-dix. Le deuxième s'inscrit dans une volonté de rationalisation des coûts d'hospitalisation au cours des deux décennies suivantes.

Entre 1960 et le début des années quatre-vingt, les hôpitaux psychiatriques sortent définitivement du modèle asilaire avec la mise en pratique de la politique de secteur (circulaire de 1960) et la réforme du cadre unique. Il s'agit de sortir d'une perspective hospitalocentriste au profit d'une ouverture sur la cité plus humaniste et moins coûteuse ; soit une réduction des temps d'hospitalisation au profit d'un suivi des patients sur des structures alternatives (centres de crise, hôpitaux de nuit, centres médico-psychologiques, etc.) et à domicile ${ }^{41}$. La réforme du cadre unique que formaient psychiatres et infirmiers ${ }^{42}$ est une des conséquences de la politique de secteur puisque le déplacement structurel des soins va générer une modification de l'organisation du travail, un élargissement du champ des compétences professionnelles, un décloisonnement de l'offre de soins et favoriser l'émergence de nouvelles catégories professionnelles. Éducateurs, aides-soignants, psychomotriciens rejoignent les équipes infirmières. L'exercice infirmier est sanctionné par un nouveau diplôme en 1973, et les effectifs augmentent.

C'est dans les années soixante-dix que les Domiens, parce qu'ils sont français, intègrent les hôpitaux psychiatriques comme infirmiers. D'une part, ils viennent combler les besoins générés par la mise en place de la sectorisation mais surtout, ils sont une main-d'œuvre de remplacement des provinciaux venus pallier les besoins croissants des asiles parisiens depuis un siècle. Dernière vague des émigrations organisées par le bureau des migrations des départements d'outre-mer (BUMIDOM), ces Domiens sont mieux préparés que leurs aînés, et l'appui de leur réseau communautaire

41. Guy BAILlon, Les Urgences de la folie : l'accueil en santé mentale, Montréal, Gaétan Morin, 1999 ; Yves BuIn, Psychiatries : l'utopie, le déclin, op. cit. ; Robert CASTEL, L'ordre psychiatrique, l'âge d'or de l'aliénisme, Paris, Éditions de Minuit, 1976 ; Marguerite COGNET, Migrations, groupes d'origines et trajectoires : vers une ethnicisation des rapports socioprofessionnels?,op. cit.

42. Philippe GIESBERGER, Évolution historique de la formation infirmière en psychiatrie - La fin d'une histoire ?, Maîtrise, Paris XII - Val-de-Marne, Créteil, 1992; Marcel JAEGER, «Garder, surveiller, soigner. Essai d'histoire de la profession d'infirmier psychiatrique », Vie sociale et traitement, 1996 (3). 
leur permet d'accéder à de meilleurs emplois que ceux auxquels l'État les destinait jusqu'alors. Néanmoins, cette opportunité se referme rapidement au cours des années quatre-vingt. Dans le cadre de la nouvelle réforme hospitalière ils sont contraints d'accepter des emplois d'aide-soignant et d'agent de service hospitalier. Cette baisse du statut socioprofessionnel affecte tout particulièrement les femmes et, avec elles, les originaires d'Afrique subsaharienne et en grande partie celles du Maghreb qui vont devoir se partager les nouvelles catégories d'emplois qui vont se créer dans le cadre des réformes sanitaires de la décennie suivante.

\subsection{Les années 1980-1990 marquées par une injonction de réduction des dépenses}

La pression économique des années quatre-vingt constitue effectivement le deuxième temps fort de cette évolution du système sanitaire français. Les dépenses des coûts de santé ont crû de façon exponentielle. La France comme tous les pays industrialisés est mise en demeure de freiner ses dépenses de santé. La psychiatrie n'échappe pas à la règle avec une durée moyenne de séjour souvent longue, les hospitalisations coûtent cher. L'appel à mieux gérer les ressources en réduisant le nombre de lits sur site hospitalier et en redéployant une partie de ceux-ci dans de plus petites structures externes, est confirmé par la circulaire de 1990 relative à la politique de santé mentale et à la loi de réforme hospitalière de 1991 qui intègre la santé mentale dans le dispositif général de santé publique. Une logique inverse à celle du placement se met en place avec une incitation au développement d'alternatives aux hospitalisations. Parmi l'ensemble des propositions et décisions politiques, citons, entre autres, a) le IX ${ }^{\mathrm{e}}$ Plan (1984-1989) qui appelle à diminuer le nombre de lits des hôpitaux de soins généraux et de psychiatrie ; b) le rapport du Commissariat général du Plan en 1985 intitulé Développer l'emploi dans les services, qui propose la création de structures privées ou associations de soutien à domicile, et l'expérimentation des moyens de paiement spécifiques pour les ménages ; et c) les premiers travaux, dans la foulée du rapport, sur la garde d'enfants et l'aide aux personnes âgées. Dans les faits, les réformes sanitaires successives entreprises au cours des années 1980 et 1990 par les gouvernements de droite comme de gauche ont été articulées autour de trois préoccupations majeures : 1) faire baisser les coûts de santé ; 2) récupérer une fiscalité qui échappe à l'État via le travail clandestin des emplois familiaux ; 3) développer un secteur d'emplois non délocalisables.

Elles se réalisent par un transfert du sanitaire au social avec le développement de structures médico-sociales, une sous-traitance au privé et un 
soutien au maintien à domicile. Deux lois vont marquer cette politique. La première, promulguée le 29 décembre 1986, crée l'allocation de garde d'enfants à domicile. La deuxième est la loi du 27 janvier 1987 qui exonère dorénavant de charges patronales les personnes âgées de plus de 70 ans, et celles à charge d'un enfant handicapé, qui ont recours aux services d'une aide à domicile. L'État promeut cette politique par tout un système d'incitations en direction des ménages (simplification des contrats de travail pour les particuliers employeurs avec le chèque emploi-service, devenu depuis le chèque emploi-service universel, aides financières sous forme d'allocations, exonérations de charges sociales, déductions fiscales, et plus récemment de crédits d'impôts). Dès le recensement de 1990, le secteur des services domestiques et d'aide à domicile, enregistre une hausse de ses effectifs (+5\%). En 1991, Martine Aubry, alors ministre du Travail, met en œuvre une politique globale de développement des « emplois familiaux ${ }^{43} »$.

En psychiatrie, la désinstitutionnalisation scinde l'activité de soin en deux pôles : un travail sur des structures légères orienté vers une psychiatrie active (centre de crise, centre d'accueil et de court séjour) et un travail dans des services de long séjour où demeurent les patients difficiles à réinsérer ou justifiant d'une prise en charge en milieu adapté (maison d'accueil spécialisée, famille d'accueil, maison de retraite médicalisée, établissement d'hébergement pour personnes âgées dépendantes, etc.). C'est dans ces lieux et nouveaux contextes d'exercice des soins que vont s'accroître les emplois d'AMP, d'assistante familiale, d'auxiliaire de vie, etc. Ces nouveaux emplois constituent un excellent exemple de l'implication des politiques de l'État dans l'exploitation des femmes issues des classes populaires et/ou issues de l'immigration.

La réorganisation globale des soins et services à la personne pour l'assister dans les actes de la vie quotidienne et domestique (que celle-ci soit maintenue à son domicile où placée dans des structures médico-sociales qui se sont diversifiées) passe aussi par une nouvelle répartition de l'offre de prestations entre les secteurs public, associatif et privé marchand. Aux côtés des contrats d'emploi de la fonction publique, et des emplois de gré à gré, où la personne se fait l'employeur de celle qui l'aide, se développent d'autres types de contrat de travail. Mais parce que l'aide aux activités de la vie quotidienne et domestique admet une certaine porosité entre ce qui est de l'ordre du soin et ce qui est de l'ordre de l'entretien et de l'hygiène de la personne et de son environnement, le problème des res-

43. Lise CAUSSE, Chantal LABRuYÈre et Christine FournIER, Les aides à domicile. Des emplois en plein remue-ménage, op. cit. 
sources humaines en matière de compétences et de droit d'exercice se pose $^{44}$. L'État devra sans cesse corriger, ajuster sa politique d'emploi et les référentiels des métiers du «care » et des métiers du social. Partant, un nombre croissant de nouveaux métiers, inévitablement en concurrence, vont se mettre à fourmiller ${ }^{45}$. Aujourd'hui, on compte près d'une vingtaine de diplômes avec les aides médico-psychologiques, les auxiliaires de vie sociale, les techniciennes de l'intervention sociale et familiale, les assistantes de vie, les employées familiales polyvalentes, les aides à domicile, etc.

L'ensemble de ces réformes à visée de rationalité économique a, par ailleurs, contribué à dégrader les conditions de l'exercice soignant dans l'ensemble du secteur public hospitalier. Si les services les plus prestigieux, aux plateaux techniques les plus sophistiqués, recrutent encore sans trop de problèmes les infirmières et aides-soignantes dont ils ont besoin, il n'en va pas de même pour les services de médecine générale, de psychiatrie et de gériatrie ${ }^{46}$. Pour pallier leurs besoins de personnel soignant, les hôpitaux accroissent, dans les années 2000, leur recours aux agences d'intérim où les migrantes sont nombreuses 47 .

Cette logique comptable en santé, qui s'accompagne pour les immigrants, et surtout pour les femmes, d'un recul du statut professionnel, d'une plus grande précarité, nous la retrouvons au Québec au début des années 1990 avec la mise en place du « virage ambulatoire ${ }^{48} »$.

\subsection{Le virage ambulatoire du Québec : mise en ouvre d'une politique d'externalisation}

Les soins et services à domicile au Canada vont, selon les provinces, prendre des allures organisationnelles plus ou moins gérées et assurées par le système public de santé. Le Québec est une des provinces où les

44. François-Xavier DEVETTER, Florence JANY-CATRICE et Thierry RIBAULT, Les services à la personne, op. cit.; Marcel JAEGER, L'articulation du santaire et du social. Travail social et psychiatrie, Paris, Dunod, 2006.

45. Valérie LANSIAUX, «Le métier d'aide médico-psychologique. Comment former des professionnels dans un contexte concurrentiel ? , TEF - Travail-Emploi-Formation, 2008 (8), p. 50-58.

46. ONI, « Démographie infirmière », Dossier de presse de l'Odre national des infirmiers, 2009, p. 10-13.

47. Bernard BROUSTET, «L'Appel médical dopé par la pénurie de personnel soignant », Les Échos, p. 24, 7 nobvembre 2001 :

http://archives.leséchos.fr/archives/2001/leséchos/18525-97-ECH.htm

48. Denyse Côté et Guilhème PÉRODEAU, Le Virage Ambulatoire. Défis et enjeux, op. cit. 
possibilités d'accueil dans ce système fut le plus développé au cours des années soixante. En 1979, le gouvernement québécois adopte une politique cadre et cible deux types d'organisation pour assurer les soins et services à domicile. Point d'entrée du dispositif, les CLSC agissent en priorité (les autres établissements publics tels que les centres de jour, hôpitaux, etc. devant leur être complémentaires). Viennent ensuite les ressources de la collectivité et parmi elles les familles, les bénévoles et les organismes communautaires.

Dans la décennie suivante, la crise de l'État-providence et la réorientation économique des systèmes de santé publics des pays industrialisés se sont étroitement conjuguées et se sont traduites par la désinstitutionnalisation massive des personnes âgées, de celles ayant des incapacités motrices et des malades mentaux. Sous la pression de celle-ci, la demande de soins et de services à domicile a crû beaucoup plus rapidement que l'offre du secteur public, conduisant les CLSC à recourir au privé ${ }^{49}$. Au début des années 1990, dans le contexte de la réforme Côté (1994), le ministère de la Santé et des Services sociaux entreprend la préparation d'une nouvelle politique de santé ${ }^{50}$ censée mettre à jour l'organisation des soins et services à domicile. Elle doit permettre de clarifier certains points obscurs, en particulier la part déléguée au privé et le rôle de l'allocation directe qui autorise une personne admissible au programme à acheter directement dans le privé les services dont elle a besoin. Des consultations et divers rapports naîtra le cadre de référence de 1994 qui n'éclairera pas pour autant la ligne de partage entre le public et le privé, ni les règles en matière d'équité, d'efficacité ou de qualité des prestations.

Il demeure que la réorientation du système de santé vers les soins à domicile a fait naître de nouveaux besoins qui ne cessent de croître sous l'influence de la demande ${ }^{51}$. Les CLSC, pour répondre à leur mission tout en respectant les contraintes économiques imposées, vont favoriser l'embauche d'auxiliaires familiaux et sociaux moins rémunérés que des infirmières auxiliaires. Parallèlement, en accord avec le gouvernement, les CLSC vont engager une importante activité de sous-traitance avec le sec-

49. Yves Vaillancourt, La Privatisation des services sociaux, Québec, Commission d'enquête sur les services de santé et services sociaux (Commission Rochon), Gouvernement du Québec, 1987.

50. MSSS, Orientations pour améliorer la santé et le bien-être au Québec, Québec, ministère de la Santé et des Services sociaux, Gouvernement du Québec, 1989; MSSS, Une réforme axée sur le citoyen, Québec, Gouvernement du Québec, ministère de la Santé et des Services sociaux, 1990.

51. Oscar FIRBANK, "Des idées à partager sur la qualité des soins à domicile », Bulletin d'information du GRASP, 2002, 1(1), 1. 
teur privé, gérant ainsi en flux tendu la demande qui, depuis, n'a cessé d'augmenter ${ }^{52}$.

Au total, dans les deux pays, les réformes de santé sur fond de rationalité économique se traduisent en partie par l'externalisation d'un certain nombre de soins et services à la personne qui favorise l'émergence d'un marché secondaire défini, par opposition au marché primaire, comme un segment du marché du travail caractérisé par la déqualification, l'insécurité d'emploi, les mauvaises conditions d'exercice, un turn-over élevé, des règles de fonctionnement et $\mathrm{d}$ 'avancement arbitraires et l'absence de soutien syndical ${ }^{53}$. Un marché qui confine une catégorie de travailleurs surnuméraires employés de façon intermittente, peu rémunérés et sans véritable protection sociale ${ }^{54}$ où les immigrants, et plus particulièrement les femmes de « couleur », sont surreprésentés.

Les immigrantes et les filles d'immigrants exerçant dans les soins et services à la personne dont nous avons retracé la situation à partir de nos enquêtes sont employées dans des conditions que l'externalisation contribue à dégrader (relégation à des statuts professionnels moins valorisés, moins rémunérés, augmentation de la précarité, perte des encadrements, des avantages sociaux, syndicaux, etc.). Ces femmes constituent une réserve de main-d'œuvre flexible, qui accède au marché du travail, mais un marché délimité par les orientations des politiques sanitaires. Leur accès aux emplois des soins et des services est fortement déterminé par leur statut de femme, par leur origine sociale et nationale et, dans le contexte actuel, par la couleur de leur peau. Il reste que la production d'une segmentation du marché et d'une division genrée et ethnicisée du travail ne tient pas qu'à la mise en œuvre de politiques sanitaires face à un marché

52. Sophie LAFOREST et Marie AMZALlAG, «Le recours à la sous-traitance pour l'offre de services d'aide à domicile par les CLSC de la région de Montréal », 2002, from www.geronto.org/frclscrenecassin/bienvieillir/fevrier2000/soustraitance.htm ; Yves VAILLANCOURT, «Le modèle québécois de politiques sociales et ses interfaces avec l'union sociale canadienne », Enjeux publics, 2002, 3(2), p. 52 ; Yves VAILlanCOURT (avec la collaboration de Christian JETTÉ), Vers un nouveau partage des responsabilités dans les services sociaux de santé : Rôles de l'État, du marché, de l'économie sociale et du secteur informel (Cahier $n^{\circ}$ 97-05, 1997), université du Québec à Montréal, Laboratoire de recherche sur les pratiques et les politiques sociales (LAREPPS).

53. Michael J. PIORE \& Suzanne BERgER, Dualism and Discontinuity in Industrial Societies, Cambridge University Press, 1980.

54. Robert CASTEL, Métamorphoses de la question sociale. Une chronique du salariat, Fayard, 1995 ; André GoRTZ, Les métamorphoses du travail. Quête de sens : critique de la raison économique, Paris, Éditions Galilée, 1988 ; Odile MERCKLING, Immigration et marché du travail. Le développement de la flexibilité en France, L'Harmattan, 1998 ; Saskia SASSEN, La ville globale : New York, Londres, Tokyo, op. cit. 
mondialisé qui s'auto-régulerait. Elle tient dans les faits tout autant aux politiques migratoires avec lesquelles elle s'agence astucieusement pour renforcer les rapports de genre traditionnels et confiner les femmes ethnicisées dans ces emplois. C'est cette historicité combinée que nous montrerons dans ce dernier point.

\section{Les programmes particuliers des politiques migratoires françaises et canadiennes}

\section{1. Importer la main-d'euvre dont la France a besoin : de la politique du BUMIDOM des années 1960 à la politique de l'immigration «choisie » des années 2000}

Nombre d' «Antillaises » que nous avons rencontrées dans le cadre de la recherche menée en France, sont arrivées en métropole via le Bureau pour le développement des migrations des départements d'outre-mer (BUMIDOM). Ceux qui n'étaient pas venus directement sous ce programme étaient en fait des frères, des sœurs ou les enfants de ceux venus avec le BUMIDOM. Créé en 1963, cet organisme est chargé d'organiser l'émigration des originaires des DOM vers la métropole en recrutant les candidats à l'émigration, en assurant leur sélection professionnelle et en gérant leur départ et leur arrivée en métropole.

Cette émigration de jeunes Domiens, organisée par l'État français, vise à résoudre deux problèmes tout en garantissant les intérêts économique et politique du pays. D'une part, il s'agit de trouver en métropole la maind'œuvre nécessaire au développement économique de la France en pleine croissance. D'autre part, l'État français essuie une récession économique importante dans les DOM qui, avec l'expansion démographique de ces départements et ses conséquences (risque de surpeuplement, difficultés de développement, crise de la jeunesse), alimente la montée des revendications indépendantistes et provoque une crise politique sans précédent. Envoyer, en aller simple, les jeunes Domiens en métropole où ils viendront pallier les besoins de main-d'œuvre est un choix politique plus stratégique $^{55}$.

De 1963 à 1980, le BUMIDOM a organisé environ 160000 émigrations. Une fois sur le sol métropolitain, ils bénéficient d'un régime "privilégié »- les femmes sont formées en priorité à la domesticité, aux ser-

55. Alain ANSELIn, L'émigration antillaise en France. La troisième Île (vol. 1), Paris, Karthala, 1990 ; Fred CONSTANT, «La politique française de l'immigration antillaise de 1946 à 1987 », Revue européenne des migrations internationales, 1987, 3, Les Antillais en Europe (3). 
vices à la personne et aux emplois d'entretien des services publics. L'État les place dans des emplois subalternes d'employé(e)s, d'ouvrier(e)s ou de personnel de service notamment dans les hôpitaux de l'Assistance publique $^{56}$. Il n'est pas étonnant que nous retrouvions ces femmes en plus grand nombre que les métropolitaines dans les emplois d'aides-soignantes et d'agentes de services hospitaliers. La nature du travail qui les confine dans le soin des personnes et de leur environnement passe par la répétition séculaire d'un apprentissage du rôle social des femmes et par la réactivation du rôle social des colonisés face aux colonisateurs ; des rôles vers lesquels, dès le départ, elles sont socialement conduites bien plus qu'elles ne les choisissent.

Aujourd'hui, le recrutement en France des femmes immigrantes doit s'appréhender dans le cadre des réformes que nous avons évoquées précédemment, les dernières émanant des plans Borloo 1 (2005) et 2 (2008), où le développement des soins et des services à la personne est décrit comme un secteur présentant « un potentiel de création d'emplois considérable », des emplois « non délocalisables » ${ }^{57}$ que le ministre entend soutenir en ouvrant mieux le marché aux entreprises et aux enseignes ${ }^{58}$. Le plan 1, avec la loi du 26 juillet 2005, crée l'agence nationale des services à la personne (ANSP). Ses missions seront fixées par le décret du 14 octobre 2005 qui va jouer un rôle important dans le recrutement des migrantes. Entre 2005 et 2006, plusieurs rapports en direction du gouvernement vont faire état de l'accroissement des besoins de main-d'œuvre et des risques de pénurie à brève échéance. L'étude de la DARES (Direction de l'animation de la recherche, des études et des statistiques), publiée en décembre 2005, sur les prévisions relatives aux impacts sur les emplois du

56. Fred CONSTANT, «La politique française de l'immigration antillaise de 1946 à $1987 »$, op. cit.

57. Audition publique devant l'Assemblée nationale le 17 mai 2006.

58. Si les entreprises privées sont autorisées depuis 1996 à intervenir sur le marché des soins et services à la personne, ce n'est que ces dernières années qu'elles s'y sont vraiment engagées. Devetter et ses collaborateurs (François-Xavier DEVETTER, Florence JANY-CATRICE et Thierry RIBAULT, Les services à la personne, op. cit., p. 56) rapportent qu'en 2006, on comptait 28000 emplois en contrat dans les entreprises privées. En Île-deFrance, les entreprises privées détenaient cette même année $30 \%$ des emplois. Les plus actives dans ce secteur demandent maintenant à être homologuées par l'agrément qualité qui leur permet d'intervenir et d'assurer des soins et services auprès des personnes en situation de grande vulnérabilité. Le plan Borloo, en 2005, ouvrira le marché aux enseignes (les banques, la poste, les assurances, etc.) censées venir grossir le secteur privé sur le marché des soins et services à la personne. 
départ à la retraite des baby-boomers ${ }^{59}$ prévoit un fort accroissement des emplois dans le secteur tertiaire, notamment dans le secteur de la santé et des services aux particuliers Ce sont des prévisions de croissance d'embauche de 40 à $60 \%$ d'ici 2015 par rapport aux emplois existants en $2005^{60}$.

L'immigration se présente donc comme une manne où puiser à condition cependant que l'on puisse choisir ceux dont on a besoin et que leur accès au territoire et aux emplois soit suffisamment contrôlé pour en conserver la maîtrise. La politique migratoire va en fixer les objectifs et les règles. La loi MISEFEN, en 2003, avait mis l'accent sur la maîtrise de l'immigration. Celle adoptée le 24 juillet $2006^{61}$ appuiera la nécessité «d'adapter l'immigration aux besoins économiques de la France » visant à «passer d'une immigration subie à une immigration choisie ${ }^{62}$. Pour atteindre cet objectif, non seulement la loi durcit les conditions qui «favorisent » l'immigration considérée comme « subie », mais elle met en œuvre le dispositif qui filtrera «l'immigration choisie», notamment par l'établissement de « listes de secteurs tendus où les employeurs pourront faire appel à des étrangers ${ }^{63}$. La circulaire du 29 avril 2006, valide une liste de 61 métiers connaissant des difficultés de recrutement. Un an plus tard, la liste est étendue à 150 métiers ouverts aux ressortissants des États membres de l'Union européenne (UE) dont : les métiers « d'aide-soignant, d'intervenant à domicile, d'employé de ménage à domicile et d'intervenant auprès d'enfants ». Sur ces 150 métiers, 30 sont ouverts aux ressortissants des pays tiers hors $\mathrm{UE}^{64}$. Tous les représentants des pays tiers ne sont

59. Olivier ChaRdon, Marc-Antoine Estrade et Fabien Toutlemonde, Les métiers en 2015 : l'impact du départ des générations du baby-boom, 2005 : http://www.travailsolidarite.gouv.fr/IMG/pdf/PIPS - 50.1 les Metiers horizon 2015.pdf.

60. M.E.F.I., Immigration sélective et besoins de l'économie française, Paris, ministère de l'Économie, des Finances et de l'Industrie, 2006.

61. Loi n 2006-911 relative à l'immigration et à l'intégration.

62. Brice Hortefeux, alors ministre de l'Immigration déclarait en 2007 que : « maîtriser l'immigration n'implique pas seulement la réguler, cela signifie aussi la choisir.» Déclaration du ministre publiée dans le Figaro le 01/06/07.

63. De plus, la loi conditionne l'obtention d'une carte de séjour «salarié » à l'existence d'un contrat de travail et à l'obtention préalable d'un visa long séjour, et parallèlement, elle institue une carte Compétence et talents valable trois ans et renouvelable. Enfin, le principe de la régularisation systématique après 10 ans de présence sur le territoire est supprimé. Cf. Politiques publiques. La politique d'immigration. Chronologie : l'histoire de l'immigration en dates, La Documentation française :

http://www.vie-publique.fr/politiques-publiques/politique-immigration/chronologieimmigration/

64. Cf. Comité interministériel de contrôle de l'immigration (CICI) du 7 novembre 2007, sur le fondement de l'article L.313-10 du CESEDA. Pour ces deux listes, la situa- 
toutefois pas logés à la même enseigne. À côté de la liste de 30 métiers, la France a signé des accords bilatéraux « de gestion concertée des flux migratoires et de développement » avec plusieurs pays du Sud (Sénégal, Gabon, Congo, Bénin, Tunisie, Île Maurice et Philippines). Leurs ressortissants peuvent accéder à certains emplois de la liste réservée aux membres de l'UE; des emplois, entre autres, « de nature sanitaire et sociale ${ }^{65}$ ». Le 12 juin 2008, Brice Hortefeux, ministre en charge de l'immigration, Laurent Wauquiez, secrétaire d'État à l'Emploi et Laurent Hénart, président de l'Agence nationale des services à la personne, signent une convention pour «faciliter » un accès rapide aux emplois de services à la personne pour les migrantes qui arrivent en situation régulière et signent un contrat d'accueil et d'intégration. Le dispositif prévoit que dès l'entretien, auquel depuis 2007 sont soumis tous les migrant(e)s sur les plates-formes d'accueil, les auditeurs sociaux effectuent un « repérage des niveaux de formation et de qualification » avant de les diriger vers les offres d'emploi. Ce dispositif est relayé par des associations comme France Terre d'Asile avec des programmes « mobilité-emploi » ${ }^{66}$.

Il reste que les entrées sur le territoire et les durées de séjour accordées sont sérieusement encadrées, et la circulaire IMI/N/07/00011/C prévoit que :

«Lors du renouvellement de l'autorisation provisoire de travail (APT), le cas échéant, il importe de vérifier que les conditions de délivrance de l'autorisation initiale ont bien été respectées (identité de métier, identité des conditions de rémunération, identité d'employeur dans le cas d'une APT). À cet égard, s'il apparaît que l'intéressé a changé de métier avant le renouvellement de son autorisation

tion de l'emploi n'est pas opposable ; autrement dit, ainsi que le précise la circulaire : «l'employeur n'est pas tenu de rechercher préalablement des candidats sur le marché du travail national et, a fortiori, de justifier de telles recherches auprès de l'administration du travail ». Cette même circulaire propose une quatrième liste de métiers en tension pour chacune des régions métropolitaines de France mais stipule que ces métiers «ne sont accessibles sans que soit opposable la situation de l'emploi que dans certaines régions ». Cette liste devant être complétée pour les DOM (Circulaire n NOR : IMI/N/07/00011/C relative aux autorisations de travail délivrées aux ressortissants des nouveaux États membres de l'Union européenne pendant la période transitoire et des États tiers, sur la base de listes de métiers connaissant des difficultés de recrutement).

65. Accord France-Île Maurice relatif au séjour et à la migration circulaire de professionnels (ensemble deux annexes), signé à Paris, le 23 septembre 2008. Consultable sur le site du GISTI : http://www.gisti.org/spip.php?article1595

66. Marguerite COGNET et Sara TOMEÏ, « Quand la politique d'immigration choisit des femmes : balai ou gant de toilette ? », presenté à "Migrations humaines et circulations des ressources. La santé en temps de mondialisation », Paris, 24-25 septembre 2009; Sara TOMEÏ, Les discriminations dans l'emploi. L'insertion des réfugiés par le dispositif PRIM de France Terre d'Asile, Master, université Paris Diderot, Paris, 2009. 
de travail et que l'activité qu'il exerce ne relève pas de la liste des métiers en tension, le critère de la situation de l'emploi sera opposable. »

Le travailleur ressortissant d'un pays tiers, qui entre au titre de la liste des 30 métiers en tension, reçoit une carte de séjour temporaire salarié si le contrat de travail proposé est d'une durée au moins égale à un an, ou une carte de séjour travailleur temporaire si le contrat est d'une durée inférieure ${ }^{67}$. Dans le cas des accords bilatéraux, les migrants peuvent se voir octroyer un titre de séjour leur permettant de rester jusqu'à 15 mois. Cette organisation de l'immigration choisie pour des métiers « en tension » rejoint l'analyse d'Odile Merckling qui, à propos des femmes immigrées, disait qu'elles devenaient les salariées de la flexibilité accrue dans le secteur des services. Par ailleurs, le système de la double liste, dans un contexte d'élargissement de l'UE à l'Est, met les migrantes déjà surexploitées dans une situation de concurrence entre elles, une concurrence particulièrement déloyale pour les migrantes des pays tiers ${ }^{68}$.

\subsection{La sélection historique des immigrants pour les besoins du marché canadien}

Le Canada, avec une histoire de peuplement et une politique d'intégration des migrants assez différentes de la France, n'en présente pourtant pas moins des similitudes intéressantes en matière de politique migratoire. D'une manière générale, nous pouvons retenir que la politique d'immigration au Canada est étroitement liée au besoin du marché. Cette politique est historiquement une politique sélective qui, après avoir longtemps formellement privilégié le critère de l'origine, a, depuis 1967, opté pour le critère économique (système de points). Nous nous concentrerons ici sur la période récente et plus spécifiquement sur un des programmes de cette politique dans la mesure où plusieurs des immigrantes que nous avons rencontrées ont raconté avoir immigré sous le programme des aides aux familiaux résidants (PAFR).

$\mathrm{Si}$, a priori, les femmes peuvent immigrer au Canada sous divers titres, c'est en général sous une étiquette qui valorise moins leur valeur personnelle et économique que celle des hommes. Ainsi, elles sont plus nombreuses $(62 \%)$ que les hommes à entrer au titre du regroupement familial. Elles sont quatre fois moins nombreuses à agir au titre de requérant prin-

67. Annexes de la circulaire du 25 juin 2008 relative à l'organisation de l'immigration professionnelle (NOR : IMI/G/08/00029/C).

68. Odile MERCKLING, «L'emploi des femmes étrangères et issues de l'immigration », op. cit.; Odile MERCKLING, Emploi, migration et genre. Des années 1950 aux années 1990, op. cit. 
cipal dans les catégories des immigrants indépendants. Enfin, on les retrouve plus nombreuses sous les programmes spéciaux (demandeurs non reconnus au statut de réfugié, individus visés par une mesure de renvoi à exécution différée, retraitées, etc.) au sein desquels le programme des aides familiaux résidants joue un rôle spécifique. Instauré en 1992, alors que le système de santé est en pleine restructuration, ce programme fait suite au Programme pour les employés de maison étrangers (PEME) qui succède lui-même à d'autres programmes gouvernementaux en matière de politique d'immigration des femmes destinées aux services domestiques depuis les années $1920^{69}$.

Mais dans les années 2000, le PAFR ne vise plus seulement des domestiques et des gardiennes d'enfants. Il sert au recrutement des personnes capables de dispenser des soins aux personnes âgées et aux handicapés. Prétendre immigrer dans le programme requiert un niveau de scolarité équivalent aux études secondaires canadiennes et une formation spécifique «en puériculture, en gérontologie, en pédiatrie ou dans le domaine des premiers soins » de « 6 mois, à temps plein, ou avoir acquis, selon les provinces, 6 à 12 mois d'expérience dans un emploi rémunéré dans ce domaine ». Ce programme d'immigration qui a sans doute permis à nombre de femmes de s'installer au Canada, s'est surtout profondément modifié en 1973, au moment où les vagues de jeunes européennes s'étaient taries pour faire place aux migrantes des pays du Sud de l'hémisphère, assurément «un peu moins blanches ». À compter de cette époque, les immigrantes sous ce programme ne pourront être reçues que sous le titre de travailleuses temporaires ${ }^{70}$. La possibilité de demander un visa de résidence permanente ne sera pas possible avant deux ans et devra répondre à des contraintes spécifiques. La première est d'être soumise à l'obligation d'effectuer deux ans de travail comme aide familiale sur une période de 3 ans dans des conditions de travail qui deviennent drastiques : l'employée doit, sous peine d'exclusion, habiter dans la résidence de son employeur. Même s'il est précisé qu'aucune disposition du contrat ne doit contrevenir à la législation

69. Myriam BALS, Les domestiques étrangères au Canada, esclaves de l'espoir, op. cit. ; Marie-Hélène VALLÉE, «L'immigration féminine au Canada durant les années 1920 : la mise en place d'une politique fondée sur le "genre", op. cit.

70. Myriam BALS, Les domestiques étrangères au Canada, esclaves de l'espoir, op. cit.; Patricia DAENZER, Regulating Class Privilege, Immigrant Servants in Canada, 1940s1990s, Toronto, Canadian Scholars Press Inc., 1993 ; Louise LANGEVIN et Marie-Claire BelleAu, «Le trafic des femmes au Canada : une analyse critique du cadre juridique de l'embauche d'aides familiales immigrantes résidantes et de la pratique des promises par correspondance », Ottawa, Condition féminine Canada, 2000 ; Tanya SCHECTER, Race, Class, Women and the State, the Case of Domestic Labour, Montreal, Black Rose Books, 1998. 
du travail tant en termes de durée journalière que de conditions d'exercice, les cas d'abus sont légion ${ }^{71}$. L'aide familiale n'a pas le droit d'exercer un autre emploi et ne peut travailler que pour l'employeur qui est nommé sur son permis de travail. C'est à elle de payer tous les frais inhérents à sa demande (examen médical, passeport) ainsi que ses frais de voyage, à moins « que son employeur n'offre de les payer ». Au-delà des obligations formelles, quand ces femmes sont hispanophones, elles sont sollicitées par les familles pour enseigner cette langue aux enfants ; service donné à titre gracieux qui a comme effet pervers de retarder leur propre apprentissage du français ou de l'anglais. Le gouvernement se dédouane de tout abus en précisant qu'il n'est pas une des parties signataires du contrat. C'est à l'employée qu'il incombe de se familiariser avec les lois qui s'appliquent dans son cas et de veiller à ses propres intérêts. Par ailleurs, la survenue d'un problème de santé grave pour la requérante, son conjoint ou l'un de ses enfants à charge est une des conditions d'inadmissibilité même après 2 ans effectués en emploi. Au bout de ces embûches, celles qui réussissent à obtenir le visa de résidente permanente sont dès lors considérées «compétentes » pour se faire embaucher comme auxiliaires familiales. Certes, elles y trouveront de meilleures conditions de travail que dans la position asservie de domestique mais leur horizon y restera limité.

La pérennité d'un tel programme d'immigration au Canada a de quoi étonner surtout lorsque l'on sait que les emplois qu'il vise sont soumis aux mêmes règles de protection du travail que tous les emplois sur le marché : le Centre de ressources humaines du Canada (CRHC) devra s'assurer qu'aucun Canadien, résident permanent, ou autre travailleur temporaire déjà au Canada ne soit assez «qualifié et disponible » pour accepter l'offre d'emploi. Faut-il en déduire qu'aucun citoyen ou résident canadien n'aurait cette qualification et cette disponibilité ? Sans doute que non. En revanche, il est licite de penser que le maintien de ce programme d'immigration s'inscrit dans une logique qui «naturalise » la place des femmes dans le prendre soin et légalise celle des immigrantes dans une «citoyenneté de seconde zone ». Une logique qui les assigne aux tâches les plus dévalorisées et les plus dévalorisantes des soins et des services aux marges du marché primaire du travail, que ce soit au titre de domestique de maison ou à celui d'auxiliaire familiale d'agences de soins et services à domicile puisque c'est pour cela qu'on leur ouvre la porte. Le «marché » des femmes immigrantes au Canada, vise l'exploitation de la force de travail de ces femmes dans un rapport inégalitaire qui les maintient

71. Myriam BALS, Les domestiques étrangères au Canada, esclaves de l'espoir, op. cit. 
confinées dans des emplois délaissés par les Canadiennes qui, dès lors, ont le privilège de pouvoir s'adonner à d'autres tâches sur un marché primaire qui leur offre des conditions de travail bien différentes ${ }^{72}$.

\section{Conclusion}

Au Canada comme en France, le recours aux immigrantes pour des postes en santé ne concerne pas n'importe quelle catégorie d'emploi. Ce sont celles qui sont les moins valorisées socialement, les moins bien rétribuées, tout en étant celles dont les conditions quotidiennes d'exercice sont souvent physiquement très éprouvantes. L'hygiène des personnes et de leur environnement n'y apparaît jamais véritablement comme un travail, du moins comme une forme de travail méritant la reconnaissance d'un certain savoir technique, a fortiori théorique. Au mieux, elle renvoie à des tâches qui font à la fois partie du rôle social des femmes, de ce qu'elles « ont à faire » en termes de rôle du genre féminin et à celles que, dans l'histoire coloniale, les femmes des colons ont délégué aux femmes colonisées générant la figure des «nounous » dont, rappelle Jules Falquet, les compétences maternelles sont doublement naturalisées puisque à la fois femmes et issues de cultures valorisant la fécondité et la maternité ${ }^{73}$.

Parce que les tâches qui relèvent des soins et services continuent d'être pensées comme relevant de la «compétence naturelle» des femmes et que les femmes des pays occidentaux les plus favorisés, ne cèderont pas leur place gagnée de haute lutte sur le marché du travail, ce sont aux immigrantes que ces sociétés recourent, dans des conditions qui n'ont pas cessé de se dégrader depuis que ces immigrantes sont des femmes du Sud. Les politiques sanitaires et les politiques migratoires sélectives structurent bien les emplois et organisent la division du travail. Leur combinaison, nolens volens, stratifie et hiérarchise cette division sur des lignes de partage par

72. Myriam BALS, Les domestiques étrangères au Canada, esclaves de l'espoir, op. cit. ; Marilyn BARBER, Les domestiques immigrantes au Canada, Ottawa, 1991.

73. Jules FALQUET, «Repenser les rapports sociaux de sexe, de classe et de "race" dans la mondialisation néolibérale », Cahiers du CEDREF, 2009 : http://www.cedref.univparis7.fr; Arlie Russel HochsCHILD, «Global Care Chains and Emotional Surplus Value », in William Hutton \& Anthony GidDENS (eds.), On the Edge : Living with Global Capitalism, New York, Free Press, 2000 ; Arlie Russel HocHSCHILD, «Love and Gold», in Barbara EHRENREICH \& Arlie Russel Hochschild (eds.), Global Women: Nannies, Maids and Sex Workers in the New Economy, New York, Henry Holt and Company, 2002 ; Éléonore KofmAn, "Genre, migrations, reproduction sociale et Welfare State », op. cit.; Saskia SASSEN, La ville globale: New York, Londres, Tokyo, (D.-A. Canal, Trans.), Paris, Descartes \& Cie, 1996 ; Marie-Hélène VALLÉE, «L'immigration féminine au Canada durant les années 1920 : la mise en place d'une politique fondée sur le "genre" », op. cit.; Sylvia WALBy, Gender Transformations, London, Routledge, 1997. 
le sexe et l'origine. Le processus invisible que soutiennent ces politiques articulées est la clé de la naturalisation genrée et ethnicisée de cette stratification hiérarchique. Elles fondent, renforcent et légitiment les représentations à l'endroit de ces femmes, les figent dans le sens commun en agrégeant l'occupation des emplois et les compétences au genre et à l'ethnicité. De cela, au-delà des migrantes, leurs filles héritent, malgré elles et leurs compétences réelles, desdites dispositions «naturelles». Établies sur un ordre sexiste et raciste, les politiques sanitaires et les politiques migratoires structurent à long terme les rapports sociaux sur ces lignes.

Tant au Québec qu'en France, les femmes que nous rencontrons disent combien leur quotidien de travail peut être difficile et ce, même si elles évoquent très majoritairement la « richesse » du contact humain. Être employées de gré à gré ou travailler dans une organisation impliquent des contraintes différentes, mais dans un cas comme dans l'autre, les femmes peuvent se sentir exposées et maltraitées. Les employées des organisations publiques ou parapubliques vivent difficilement le poids d'une institution qui ne les reconnaît quasiment pas. Beaucoup disent avoir le sentiment d'être méprisées par leurs supérieurs et les autres corps professionnels. Quand elles travaillent de gré à gré, elles gagnent en autonomie mais travaillent de façon isolée et vivent une plus grande précarité. Plusieurs d'entre elles évoquent également les difficultés qu'elles peuvent rencontrer avec les exigences et l'arbitraire de leurs employeurs (qui sont souvent d'autres femmes mais d'une autre classe ou/et d'une autre origine). Le travail pour le compte des agences privées à but lucratif introduit un marché secondaire du fait du jeu de la concurrence marchande. Les employées perdent la sécurité des conditions des contrats de travail qu'elles peuvent avoir dans les institutions publiques et parapubliques sans avoir pour autant la contrepartie de l'autonomie qu'elles ont dans les contrats gré à gré. En France, l'accroissement de la part que vont prendre les entreprises privées et l'arrivée des enseignes en France selon le cadre défini dès le premier plan Borloo, pourraient bien aggraver la situation des femmes employées de gré à gré ou par le biais d'associations ainsi que nous l'avons montré pour le Canada où les agences privées sont très implantées ${ }^{74}$.

74. Marguerite COGNET, «Family and Social Assistants : Satisfied but Critical of their Working Conditions », Bien-vieillir, Bulletin du CLSC René Cassin, 2003 ; «Politiques d'immigration canadiennes et femmes d'ailleurs dans les emplois dans le secteur des soins et des services à domicile ", in Francine SAILlant et Manon Boulianne (éds.), Transformations sociales, genre et santé. Perspectives critiques et comparatives, Québec-Paris, Presses de l'université Laval et L'Harmattan, 2003, p. 183-205 ; Marguerite CoGNET, «L'accès à l'emploi dans les services de santé : l'effet de la dimension ethnique. Étude du groupe des auxiliaires familiaux et préposés aux bénéficiaires à Montréal », op. cit.; 
Dans un article récent, Éléonore Kofman invitait à «prendre en compte le rôle de l'État dans la production de l'offre et de la demande pour ce qui concerne le travail de care, et ne pas supposer que c'est le marché seul qui provoque le transfert de travail entre des foyers tout autour du globe $^{75} »$. Elle ajoutait que « les lois visant à réguler l'immigration structurent l'offre de travail par des politiques d'immigration sélectives », et que de celles-ci « résulte un système complexe de stratifications liées aux rapports sociaux de classe, de sexe et de "race", fondé sur l'articulation des qualifications obtenues, du mode d'entrée dans le pays d'immigration et de la nationalité : la concurrence mondiale, pour attirer les migrant(e)s les plus qualifié(e)s, a rendu les pays d'immigration particulièrement bienveillants à leur égard». Nos analyses prolongent la pensée de Kofman en montrant que la face cachée de cette bienveillance à l'égard de l'immigration convoitée est la part, dans l'ombre, réservée aux femmes des pays du Sud qui n'ont pas vraiment d'autre choix que de venir au chevet des gens des pays riches du Nord dans des emplois dévalorisés et dévalorisants.

Université Paris VII - Denis Diderot - URMIS

$* \quad * \quad *$

"Quand l'infirmière est noire. Les soins et services de santé dans un contexte raciste », Face à Face, 2004 (6), p. 10-21.

75. Éléonore KOFMAN, «Genre, migrations, reproduction sociale et Welfare State », op. cit., p. 114. 